\title{
Resistive-wall wake and impedance for nonultrarelativistic beams
}

\author{
Frank Zimmermann* \\ CERN, AB Department, 1211 Geneva 23, Switzerland \\ Katsunobu Oide ${ }^{\dagger}$ \\ KEK, 1-1 Oho, Tsukuba-shi, Ibaraki-ken 305, Japan \\ (Received 15 February 2004; published 9 April 2004)
}

\begin{abstract}
The usual formulas for the resistive-wall wake field are derived considering ultrarelativistic beams, traveling at the speed of light. This simplifies the calculation, and it leads to a cancellation between electric and magnetic fields. However, for proton beams below $10 \mathrm{GeV}$ and for many heavy-ion beams, the velocities may significantly differ from the speed of light. In this paper, we compute the longitudinal and transverse wake fields for velocities smaller than $c$ and examine under which conditions nonrelativistic effects become important. We illustrate our results by a few examples.
\end{abstract}

DOI: $10.1103 /$ PhysRevSTAB.7.044201

PACS numbers: 29.27.Bd, 29.20.Dh, 41.75.-i

\section{INTRODUCTION}

Presently several accelerators are under construction which aim to produce intense proton or ion beams at energies around $1 \mathrm{GeV}$, for example, the Spallation Neutron Source (SNS) [1] and the Japan Proton Accelerator Research Complex (J-PARC) [2]. Also the Large Hadron Collider (LHC) beam in the CERN Proton Synchrotron (PS) and in the PS booster [3] is not or only moderately relativistic. One possible performance limitation for these types of beams may arise from the resistive-wall impedance.

The conventional treatment of the resistive-wall wake field considers an ultrarelativistic beam; see, e.g., Refs. $[4,5]$. Only few papers have attempted to treat the general case. A rare and early example is Ref. [6], but in the ultrarelativistic limit its wake field does not reduce to the conventional form. An expression for the longitudinal impedance due to space charge and the resistive wall for a beam of finite transverse size can be found in Ref. [7]. A general formalism for computing impedances of nonrelativistic beams, including resistive-wall boundaries, was given by Gluckstern [8].

Our results differ from the earlier papers in that we (i) derive an explicit nonrelativistic correction of the resistive-wall impedance, (ii) start from the exact solution of Maxwell's equations inside the wall for a circular geometry, (iii) derive the associated Green-function wake fields, (iv) use an alternative method for calculating the transverse impedance, and (v) examine implications for several operating and planned accelerators.

This paper is organized as follows. The terms wake field and impedance are defined in Sec. II, for the general nonultrarelativistic case. In Sec. III we derive the nonultrarelativistic longitudinal monopole impedance and

\footnotetext{
*Electronic address: frank.zimmermann@cern.ch

${ }^{\dagger}$ Electronic address: katsunobu.oide@kek.jp
} erated by a pointlike source - through first order in the skin depth and second order in $1 / \gamma$. In Sec. IV we repeat this calculation for the more complicated case of the dipole mode. We here obtain the transverse impedance and the transverse Green-function wake field. Section V compares some of our results with earlier calculations. Section VI investigates various limits of our treatment, most of which are related to the breakdown of Ohm's law under certain conditions. In Sec. VII we apply the wakefield expressions derived in this paper to four example accelerators, in order to unveil the significance of the nonrelativistic correction for each case. The main conclusions are summarized in Sec. VIII.

\section{DEFINITIONS}

Some authors reserve the terms wake field and impedance exclusively for ultrarelativistic beams, where the longitudinal monopole and the transverse dipole wake fields have special features, e.g., they are equal to zero ahead of the source by virtue of causality and they do not depend on the transverse position of the test particle nor on the beam energy. However, we can easily generalize the concepts of wake field and impedance to beams of arbitrary velocity. As in the ultrarelativistic case, also for the general nonultrarelativistc situation we may define the longitudinal monopole wake field $W_{0}^{\prime}$, the longitudinal dipole wake field $W_{1, \|}$, and the transverse dipole wake field $W_{1, \perp}$ via the fields experienced by a charged test particle propagating a distance $z$ behind a source with monopole moment (charge) $q$ or dipole moment $q a$ (where $a$ has units of length), respectively. More precisely, the general Green-function wake fields per unit length can be expressed in analogy to Eq. (2.50) of Ref. [4] as 


$$
\begin{aligned}
-E_{s}^{(0)} & =q W_{0}^{\prime}(z, r, \gamma), \\
-E_{s}^{(1)} & =q a r W_{1, \|}(z, r, \gamma)\left(\vec{u}_{r} \cos \varphi-\vec{u}_{\varphi} \sin \varphi\right), \\
-\left(\vec{E}+\vec{u}_{s} \times \vec{B}\right)_{\perp}^{(1)} & =q a W_{1, \perp}(z, r, \gamma)\left(\vec{u}_{r} \cos \varphi-\vec{u}_{\varphi} \sin \varphi\right),
\end{aligned}
$$

where $u_{s}, u_{r}$, and $u_{\varphi}$ denote unit vectors in cylindrical coordinates with $u_{s}$ pointing in the direction of beam propagation, the coordinates $r, \varphi$, and $z$ describe the position of the test particle, the superindices "( 0$)$ " or "(1)" distinguish monopole and dipole wakes, and the dipole-moment source density has the assumed angular dependence $\cos \varphi$. In the ultrarelativistic limit, the wake functions $W_{0}^{\prime}, W_{1, \|}$, and $W_{0, \perp}$ introduced above are independent of $r$ and $\gamma$ and equal to the functions $W_{0}^{\prime}(z)$, $W_{1}^{\prime}(z)$, and $W_{1}(z)$ of Ref. [4].

As in the ultrarelativistic case, with each general Green-function wake a frequency-dependent impedance is associated via a Fourier transform. For example the wake field $W_{0}^{\prime}(z, r, \gamma)$ and its associated longitudinal monopole impedance $Z_{\|}(\omega, r, \gamma)$ are related by

$$
W_{0}^{\prime}(z, r, \gamma)=\frac{1}{2 \pi} \int_{-\infty}^{\infty} d \omega e^{i \omega z / v} Z_{\|}(\omega, r, \gamma)
$$

and

$$
Z_{\|}(\omega, r, \gamma)=\int_{-\infty}^{\infty} \frac{d z}{c} e^{-i \omega z / v} W_{0}^{\prime}(z, r, \gamma)
$$

\section{LONGITUDINAL WAKE}

\section{A. The problem}

We consider a beam pipe of radius $b$ with conductivity $\sigma$, independent of angular frequency $\omega$. Also we assume that the beam charge line density $\lambda_{b} \exp (i k s-i \omega t)$ travels at the center of the beam pipe with frequency $\omega=v k$ and velocity $v<c$. Following Chao's treatment of the ultrarelativistic case [4], we introduce a new variable $z=$ $(s-v t)$. Then all quantities have the same dependence $\exp (i k z)$ on $s$ and $t$.

\section{B. Potentials and Lorentz condition}

The electric and magnetic fields are related to the scalar potential $\phi$ and the magnetic vector potential $\vec{A}$ via

$$
\begin{gathered}
\vec{E}=-\nabla \phi-\partial \vec{A} / \partial t, \\
\vec{B}=\nabla \times \vec{A} .
\end{gathered}
$$

For an arbitrary medium with relative dielectric constant $\epsilon_{r}$ and relative magnetic permeability $\mu_{r}$ AmpèreMaxwell's law is

$$
\vec{\nabla} \times \vec{B}=\mu_{0} \mu_{r} \vec{j}+\frac{\mu_{r} \epsilon_{r}}{c^{2}} \frac{\partial \vec{E}}{\partial t}
$$

or

$$
\vec{\nabla} \times(\vec{\nabla} \times \vec{A})=\frac{\mu_{r} \epsilon_{r}}{c^{2}} \frac{\partial}{\partial t}\left(-\nabla \phi-\frac{\partial \vec{A}}{\partial t}\right)+\mu_{0} \mu_{r} \vec{j},
$$

which can be rewritten as

$$
-\triangle \vec{A}+\frac{\mu_{r} \epsilon_{r}}{c^{2}} \frac{\partial^{2} \vec{A}}{\partial t^{2}}+\vec{\nabla}\left(\vec{\nabla} \cdot \vec{A}+\frac{\mu_{r} \epsilon_{r}}{c^{2}} \frac{\partial \phi}{\partial t}\right)=\mu_{0} \mu_{r} \vec{j} .
$$

Imposing the Lorentz condition

$$
\left(\vec{\nabla} \cdot \vec{A}+\frac{\mu_{r} \epsilon_{r}}{c^{2}} \frac{\partial \phi}{\partial t}+\mu_{0} \mu_{r} \sigma \phi\right)=0,
$$

the potential $\vec{A}$ fulfills the equation

$$
-\triangle \vec{A}+\frac{\mu_{r} \epsilon_{r}}{c^{2}} \frac{\partial^{2} \vec{A}}{\partial t^{2}}+\mu_{0} \mu_{r} \sigma \frac{\partial \vec{A}}{\partial t}=\mu_{0} \mu_{r}(\vec{j}-\sigma \vec{E}) .
$$

Similarly, from Gauss's law $\vec{\nabla} \cdot \vec{E}=\rho /\left(\epsilon_{0} \epsilon_{r}\right)$, and using the Lorentz condition (9) we obtain for the potential $\phi$

$$
-\triangle \phi+\frac{\mu_{r} \epsilon_{r}}{c^{2}} \frac{\partial^{2} \phi}{\partial t^{2}}+\mu_{0} \mu_{r} \sigma \frac{\partial \phi}{\partial t}=\frac{\rho}{\epsilon_{0} \epsilon_{r}} .
$$

The two wave equations (10) and (11) are well known. Together with the Lorentz condition (9), they form the starting point of our impedance calculation.

In the following we will apply the wave equations either to the vacuum inside the beam pipe, or to the beam-pipe wall, and, for simplicity, we will consider only wall materials with $\mu_{r}=\epsilon_{r}=1$.

\section{Wave equations in vacuum}

We first consider the potentials and electric fields for the vacuum region enclosed by the beam pipe, for which $\sigma=0$ and $\epsilon_{r}=\mu_{r}=0$. In case of the longitudinal wake, the only nonzero field components are $E_{s}, E_{r}$, and $B_{\varphi}$ because of symmetry, i.e., there is no dependence on $\varphi$. We can thus set $A_{\varphi}=0$ and $A_{r}=0$.

Note, for example, that if $A_{r}$ is not zero, we can make it vanish by a gauge transformation to the new $A_{r}^{\prime}$ and $\phi^{\prime}$,

$$
\begin{aligned}
& \vec{A}^{\prime}=\vec{A}-\vec{\nabla} \psi, \\
& \phi^{\prime}=\phi+\frac{\partial \psi}{\partial t},
\end{aligned}
$$

through the gauge function $\psi=\int^{r} A_{r}\left(r^{\prime}\right) d r^{\prime}$. It is easy to see that this preserves the Lorentz condition (9), since $A_{r}$ is a solution of (10) and $j_{r}=0$.

The Lorentz condition (9) relates the two nonzero components of the potential, $A_{s}$ and $\phi$, as

$$
\phi=\frac{c^{2} k}{\omega} A_{s} \text {. }
$$


Thus it is sufficient to compute $A_{s}$. We decompose the two potentials and the sources as

$$
\begin{aligned}
\phi & =\tilde{\phi} e^{i k z}, & A_{s} & =\tilde{A}_{s} e^{i k z}, \\
\lambda_{b} & =\tilde{\boldsymbol{\rho}}_{b} e^{i k z}, & j_{s} & =\tilde{j}_{s} e^{i k z} .
\end{aligned}
$$

The phasor coefficients $\tilde{\phi}_{s}$ and $\tilde{A}_{s}$ must fulfill the two wave equations

$$
\begin{gathered}
\frac{1}{r} \frac{\partial}{\partial r}\left(r \frac{\partial \tilde{A}_{s}}{\partial r}\right)-k_{r}^{2} \tilde{A}_{s}=-\mu_{0} \tilde{j}_{s}, \\
\frac{1}{r} \frac{\partial}{\partial r}\left(r \frac{\partial \tilde{\phi}}{\partial r}\right)-k_{r}^{2} \phi=-\frac{\tilde{\rho}_{0}}{\epsilon_{0}},
\end{gathered}
$$

where the parameter $k_{r}$, related to the nonrelativistic speed of the beam, is given by

$$
k_{r}=\left(k^{2}-k_{0}^{2}\right)^{1 / 2}=\frac{\left|k_{0}\right|}{\beta \gamma}>0,
$$

with $k_{0}=\omega / c$.

\section{General solution for longitudinal potential}

The right-hand sides of (15) and (16) are zero except for inside the beam. Outside the beam, the general solution to Eq. (15) is

$$
\tilde{A}_{s}=p I_{0}\left(k_{r} r\right)+q K_{0}\left(k_{r} r\right),
$$

where $I_{0}$ and $K_{0}$ denote the modified Bessel functions of 0 th order, and $q$ and $p$ are coefficients yet to be found. From (14) we also know that

$$
\tilde{\phi}_{s}=\frac{c^{2} k}{\omega}\left[p I_{0}\left(k_{r} r\right)+q K_{0}\left(k_{r} r\right)\right]
$$

Considering a right-handed coordinate $\operatorname{system}(r, \varphi, z)$, the nonvanishing electric and magnetic fields are obtained from the potential via

$$
\begin{aligned}
B_{\varphi} & =-\frac{\partial A_{s}}{\partial r} \\
& =\left[-p k_{r} I_{0}^{\prime}\left(k_{r} r\right)-q k_{r} K_{0}^{\prime}\left(k_{r} r\right)\right] \exp (i k z) \\
& =\left[-p k_{r} I_{1}\left(k_{r} r\right)+q k_{r} K_{1}\left(k_{r} r\right)\right] \exp (i k z) \\
E_{s} & =-\frac{\partial \phi}{\partial s}-\frac{\partial A_{s}}{\partial t} \\
& =-i \omega \frac{k_{r}^{2}}{k_{0}^{2}}\left[p I_{0}\left(k_{r} r\right)+q K_{0}\left(k_{r} r\right)\right] \exp (i k z) \\
E_{r}= & -\frac{\partial \phi}{\partial r}=-\frac{c^{2} k k_{r}}{\omega}\left[p I_{1}\left(k_{r} r\right)-q K_{1}\left(k_{r} r\right)\right] \exp (i k z)
\end{aligned}
$$

where we have used $I_{0}^{\prime}=I_{1}$ and $K_{0}^{\prime}=-K_{1}$.

\section{E. Source boundary}

The coefficient $q$ is determined by the source current, and the coefficient $p$ by the surface condition at $r=b$. First we compute $q$. The source term $-\mu_{0} j_{s}$ on the righthand side of (15), after integration over the transverse plane, equals $-\mu_{0} \lambda_{b} v$. The Green function of the operator on the left-hand side for a point source is $\ln r /(2 \pi)$. Hence, the potential $A_{s}$ should approach $\left[-\mu_{0} \lambda_{b} v \ln r /(2 \pi)\right]$. For small arguments, the modified Bessel function $I_{0}$ approaches 1 , whereas $K_{0}$ has the expansion $-\ln z$. By comparing the diverging term $q K_{0}$ with the required asymptotic expansion, we obtain

$$
q=\frac{\mu_{0} \lambda_{b} v}{2 \pi}
$$

\section{F. Wall boundary}

To find the missing coefficient $p$, we assume that the inside of the conducting metallic wall is characterized by the absence of a net charge, by a relative magnetic permeability, and a relative dielectric constant equal to 1 , and by Ohm's law:

$$
\begin{gathered}
\vec{j}=\sigma \vec{E}, \\
\mu_{r}=1, \\
\epsilon_{r}=1, \\
\rho=0 .
\end{gathered}
$$

Then, inside the wall the right-hand sides of (10) and (11) vanish, and the wave equations for the potentials become

$$
\begin{gathered}
\frac{1}{r} \frac{\partial}{\partial r}\left(r \frac{\partial A_{s}}{\partial r}\right)=-\left(i \mu_{0} \sigma \omega-k_{r}^{2}\right) A_{s}, \\
\frac{1}{r} \frac{\partial}{\partial r}\left(r \frac{\partial \phi}{\partial r}\right)=-\left(i \mu_{0} \sigma \omega-k_{r}^{2}\right) \phi,
\end{gathered}
$$

where $k_{r}^{2}=k^{2}-k_{0}^{2}$ as before. We note that for the more general case $\mu_{r} \neq 1$, not further considered below, the conductivity $\sigma$ and the square free-space wave number $k_{0}^{2}$, on the right sides of (28) and (29), must be multiplied by $\mu_{r}$.

We would like to rewrite this in a more compact form. To this end, we introduce the skin depth

$$
\delta_{\text {skin }} \equiv \sqrt{\frac{2}{\mu_{0} \sigma|\omega|}},
$$

and the two related parameters 


$$
\lambda_{0} \equiv \frac{1-i \operatorname{sgn}(\omega)}{\delta_{\text {skin }}}
$$

and

$$
\lambda^{2} \equiv \lambda_{0}^{2}+k_{r}^{2}=-\left(i \mu_{0} \sigma \omega-k_{r}^{2}\right)
$$

In (32), we recognize the coefficients on the right-hand sides of (28) and (29). These latter equations become

$$
\begin{gathered}
\frac{1}{r} \frac{\partial}{\partial r}\left(r \frac{\partial A_{s}}{\partial r}\right)-\lambda^{2} A_{s}=0, \\
\frac{1}{r} \frac{\partial}{\partial r}\left(r \frac{\partial \phi}{\partial r}\right)-\lambda^{2} \phi=0 .
\end{gathered}
$$

Again the solutions are 0th order Bessel functions,

$$
\begin{gathered}
A_{s}=q^{w} K_{0}(\lambda r) \exp (i k z), \\
\phi=\left[\frac{1}{\frac{\omega}{k c^{2}}+i \frac{\mu_{0} \sigma}{k}}\right] q^{w} K_{0}(\lambda r) \exp (i k z),
\end{gathered}
$$

where, in the last line, we have invoked the Lorentz condition (9); the value of $\lambda$, in (32), is chosen so that in the ultrarelativistic limit it coincides with $\lambda_{0}$ of (31). In (35) and (36), all other possible solutions of (33) and (34), namely, those involving the Bessel function $I_{0}$ or the second value of $\lambda$ permitted by (32), $\lambda \approx-\lambda_{0}$, were discarded, since for these solutions the magnitude of the Bessel functions would diverge as a function of radius $r$. This approximation applies only if the thickness of the beam pipe is large compared with the skin depth.

The longitudinal and radial electric fields in the wall are obtained from (21) and (22) as

$$
\begin{aligned}
& E_{s}=q_{s w} K_{0}(\lambda r) \exp (i k z), \\
& E_{r}=q_{r w} K_{1}(\lambda r) \exp (i k z),
\end{aligned}
$$

where

$$
\begin{gathered}
q_{s w}=-i \omega\left[\frac{\lambda^{2}}{k_{0}^{2}-\lambda_{0}^{2}}\right] q^{w}, \\
q_{r w}=\omega\left(\frac{k \lambda}{k_{0}^{2}-\lambda_{0}^{2}}\right) q^{w},
\end{gathered}
$$

and $\lambda$, defined in (32), is complex. The electric fields fulfill the 0th and 1st order modified Bessel equations,

$$
\begin{gathered}
\frac{1}{r} \frac{\partial}{\partial r}\left(r \frac{\partial E_{s}}{\partial r}\right)-\lambda^{2} E_{s}=0, \\
\frac{\partial^{2} E_{r}}{\partial r^{2}}+\frac{1}{r} \frac{\partial E_{r}}{\partial r}-\frac{E_{r}}{r^{2}}-\lambda^{2} E_{r}=0 .
\end{gathered}
$$

We note that $\lambda$, (32), can be expanded as

$$
\lambda \approx \lambda_{0}\left(1+\frac{k_{r}^{2}}{2 \lambda_{0}^{2}}\right)=\lambda_{0}\left(1+i \operatorname{sgn}(\omega) \frac{k_{r}^{2} \delta_{\text {skin }}^{2}}{4}\right),
$$

where, in the last step, we explicitly convey a nonrelativistic correction. However, since this correction term is of higher order in the skin depth, we will neglect it in our final expressions.

From (20), it follows that

$$
B_{\varphi}=\lambda q^{w} K_{1}(\lambda r) \exp (i k z),
$$

which, inserting (39), can be rewritten in terms of $q_{s w}$ as

$$
B_{\varphi}=\frac{1}{i \omega}\left(-\frac{k^{2}}{\lambda}+\lambda\right) K_{1}(\lambda r) q_{s w} \exp (i k z) .
$$

Matching the tangential fields $E_{s}$ and $B_{\varphi}$, (37) and (45), at the chamber wall, $r=b$, with those inside the beam pipe, Eqs. (20) and (21), we obtain two equations:

$$
\begin{aligned}
-i \omega k_{r}^{2}\left[p I_{0}\left(k_{r} b\right)+q K_{0}\left(k_{r} b\right)\right] & =q_{s w} k_{0}^{2} K_{0}(\lambda b), \\
i \omega k_{r}\left[-p I_{1}\left(k_{r} b\right)+q K_{1}\left(k_{r} b\right)\right] & =q_{s w}\left(\lambda-\frac{k^{2}}{\lambda}\right) K_{1}(\lambda b) .
\end{aligned}
$$

We can now solve these for the two unknown coefficients $p$ and $q_{s w}$ :

$$
\begin{aligned}
\frac{q_{s w}}{q} & =-\frac{i k_{r}^{2} \lambda \omega\left[I_{1}\left(b k_{r}\right) K_{0}\left(b k_{r}\right)+I_{0}\left(b k_{r}\right) K_{1}\left(b k_{r}\right)\right]}{k_{0}^{2} \lambda I_{1}\left(b k_{r}\right) K_{0}(\lambda b)+k_{r}\left(k^{2}-\lambda^{2}\right) I_{0}\left(b k_{r}\right) K_{1}(b \lambda)}, \\
\frac{p}{q} & =\frac{k_{0}^{2} \lambda K_{1}\left(b k_{r}\right) K_{0}(b \lambda)+k_{r}\left(\lambda^{2}-k^{2}\right) K_{0}\left(b k_{r}\right) K_{1}(\lambda b)}{k_{0}^{2} \lambda I_{1}\left(b k_{r}\right) K_{0}(b \lambda)-k_{r}\left(\lambda^{2}-k^{2}\right) I_{0}\left(b k_{r}\right) K_{1}(\lambda b)} .
\end{aligned}
$$

From (18) and (23), the complex amplitude $\tilde{A}_{s}$ of the magnetic vector potential $A_{s}=\tilde{A}_{s} e^{i k z}$ becomes

$$
\tilde{A}_{s}=\frac{\mu_{0} \lambda_{b} v}{2 \pi}\left[K_{0}\left(k_{r} r\right)+I_{0}\left(k_{r} r\right) \frac{k_{0}^{2} \lambda K_{1}\left(b k_{r}\right) K_{0}(b \lambda)+k_{r}\left(\lambda^{2}-k^{2}\right) K_{0}\left(b k_{r}\right) K_{1}(\lambda b)}{k_{0}^{2} \lambda I_{1}\left(b k_{r}\right) K_{0}(b \lambda)-k_{r}\left(\lambda^{2}-k^{2}\right) I_{0}\left(b k_{r}\right) K_{1}(\lambda b)}\right] .
$$

\section{G. Impedance}

The longitudinal impedance is closely tied to the longitudinal electric field. The latter can be computed from the longitudinal vector potential $A_{s}$ in (47) as $E_{s}=\tilde{E}_{s} e^{i k z}$ with

$$
\tilde{E}_{s}=-i c^{2} k_{r}^{2} / \omega \tilde{A}_{s}
$$

We define the impedance by Fourier transformation with respect to time $t$ and not with respect to $z$, since the frequency is a continuous variable, while the wave number $k$ does not assume any values between $-k_{r}$ and $k_{r}$.

The longitudinal impedance per unit length at frequency 


$$
\omega=c \sqrt{k^{2}-k_{r}^{2}}
$$

is related to the field $\tilde{E}_{s}(k)$ via

$$
Z_{\|}(\omega)=-\tilde{E}_{s}(k) / \bar{j}_{s}
$$

where $\bar{j}_{s}=\lambda_{b} v$, or

$$
Z_{\|}(\omega)=\frac{i Z_{0} c k_{r}^{2}}{2 \pi \omega}\left[K_{0}\left(k_{r} r\right)+I_{0}\left(k_{r} r\right) \frac{\omega^{2} \lambda K_{1}\left(b k_{r}\right) K_{0}(b \lambda)+k_{r} c^{2}\left(\lambda^{2}-k^{2}\right) K_{0}\left(b k_{r}\right) K_{1}(\lambda b)}{\omega^{2} \lambda I_{1}\left(b k_{r}\right) K_{0}(b \lambda)-k_{r} c^{2}\left(\lambda^{2}-k^{2}\right) I_{0}\left(b k_{r}\right) K_{1}(\lambda b)}\right]
$$

and $Z_{0}=\mu_{0} c=120 \pi \Omega$ denotes the vacuum impedance. Equation (51) is our general result for the impedance in the longitudinal plane. As we shall see, it contain a component related to the space charge and another related to the resistive wall as well as mixed expressions, which represent the nonrelativistic corrections to the resistivewall impedance.

For many applications, we may simplify the formula (51) by expansions and approximations. In particular, we may consider the common case that the skin depth is small compared with the beam-pipe aperture, or $|\lambda b| \gg 1$. In this limit, we can expand the Bessel functions of a complex variable $z=\lambda b$ with $|\arg (z)|<$ $\pi / 2$ as [9]

$$
\begin{aligned}
& K_{0}(z) \approx e^{-z} \sqrt{\frac{\pi}{2 z}}\left(1-\frac{1}{8 z}\right) \approx e^{-z} \sqrt{\frac{\pi}{2 z}} \\
& K_{1}(z) \approx e^{-z} \sqrt{\frac{\pi}{2 z}}\left(1+\frac{3}{8 z}\right) \approx e^{-z} \sqrt{\frac{\pi}{2 z}} .
\end{aligned}
$$

If we further assume that $k_{r} \delta_{\text {skin }} \ll 1$, from Eq. (43) we can approximate $\lambda \approx \lambda_{0}$. Then the impedance in Eq. (51) simplifies to

$$
Z_{\|}(\omega)=\frac{i Z_{0} c k_{r}^{2}}{2 \pi \omega}\left[K_{0}\left(k_{r} r\right)-\frac{[1-i \operatorname{sgn}(\omega)] c^{2} K_{0}\left(k_{r} b\right)+\delta_{\text {skin }} \omega^{2} K_{1}\left(k_{r} b\right)}{[1-i \operatorname{sgn}(\omega)] c^{2} I_{0}\left(k_{r} b\right)-\delta_{\text {skin }} \omega^{2} I_{1}\left(k_{r} b\right)} I_{0}\left(k_{r} r\right)\right],
$$

where the skin depth $\delta_{\text {skin }}$ refers to the angular frequency $\omega$.

Expanding $Z_{\|}(\omega)$ of Eq. (54) to second order in $\left(k_{r} c / \omega\right)$ and to first order in $\left(\delta_{\text {skin }} \omega / c\right)$, keeping the mixed terms proportional to $\left(\delta_{\text {skin }} k_{r}^{2}\right)$, this becomes

$$
\begin{aligned}
Z_{\|}(\omega) \approx \frac{Z_{0}}{2 \pi c}[ & \frac{[\operatorname{sgn}(\omega)-i] \omega \delta_{\text {skin }}}{2 b}\left(1-\frac{k_{r}^{2}\left(2 b^{2}-r^{2}\right)}{4}\right) \\
& \left.-i \frac{c^{2} k_{r}^{2}}{\omega} \ln \frac{r}{b}\right] .
\end{aligned}
$$

We have verified that exactly the same expansion is obtained, if we start from (51) and approximate the Bessel functions as in (52) and (53), but do not make the approximation $\lambda \approx \lambda_{0}$. Hence, the nonrelativistic corrections to $\lambda$ indeed contribute to the impedance in higher order only.

We may identify the last term in (55), which is independent of $\lambda$, with the space-charge impedance,

$$
Z_{\|, \mathrm{sc}}(\omega) \approx-i \frac{Z_{0}}{2 \pi c} \frac{c^{2} k_{r}^{2}}{\omega} \ln \frac{r}{b}
$$

The other terms, which are linear in the skin depth, describe the resistive-wall contribution including the lowest-order nonrelativistic correction:

$Z_{\|, \mathrm{rw}}(\omega) \approx \frac{Z_{0}}{2 \pi c}\left[\frac{[\operatorname{sgn}(\omega)-i] \omega \delta_{\text {skin }}}{2 b}\left(1-\frac{k_{r}^{2}\left(2 b^{2}-r^{2}\right)}{4}\right)\right]$.

If $k_{r} \neq 0$ this impedance depends not only on the longitudinal distance $z$ from the source, but also on the radial position $r$ and, via $k_{r}$, on the relativistic Lorentz factor $\gamma$.

In the ultrarelativistic limit, $k_{r} \rightarrow 0$, (57) becomes

$Z_{\|, \mathrm{rw}}(\omega) \approx k_{r} \rightarrow 0 \frac{Z_{0} \omega}{2 \pi c} \frac{[\operatorname{sgn}(\omega)-i] \delta_{\text {skin }}}{2 b}=\frac{1-i \operatorname{sgn}(\omega)}{2 \pi b \delta_{\text {skin }} \sigma}$,

which agrees with Eq. (2.77) in Ref. [4].

\section{H. Green-function wake}

From the impedance we compute the longitudinal Green-function wake per unit length as

$$
W_{0}^{\prime}(z)=\frac{1}{2 \pi} \int_{-\infty}^{\infty} Z_{\|}(\omega) e^{i \omega z / v} d \omega
$$

If the impedance has no singularity for $\omega$ in the upper complex plane, the wake is zero ahead of the source $(z>0)$. In our case, the impedances (51) and (54) have many singularities in the upper complex plane, so that the wake field in front of the source is not zero in general. This is expected, since the electromagnetic fields can propagate faster than the beam, if the latter is not ultrarelativistic. However, approximating the impedance by (55) eliminates all these singularities. In particular, under this approximation, the Green-function wake that corresponds to the space-charge impedance, Eq. (56), is proportional to the derivative of a delta function $\delta^{\prime}(z)$. For the resistive-wall impedance, in (57), we are left with a 
peculiar singularity at the origin, which is of the type $(|\omega|-i \omega) / \sqrt{|\omega|}$. A rigorous solution to performing the integral for this impedance may be found by invoking advanced techniques of complex analysis. We here present a heuristic way of computation. Namely, to fulfill causality, we move the singularity into the lower complex plane, by replacing $\omega$ with $|\omega+i \epsilon|$, where $\epsilon$ is an infinitesimal number. This implies that the wake is damped in time as $\exp (-\epsilon t)$. So we might think of $\epsilon$ as representing a small damping term, e.g., representing Ohmic heating or radiation. After introducing $\epsilon$, for $z>0$ we can close the integration contour in the upper complex plane and, without any singularity left in this half plane, the integral is zero by virtue of Cauchy's theorem. Therefore, causality is fulfilled in the ultrarelativistic limit, as it should be.
We now consider the region $z<0$, where the wake is finite. The impedance (57) is rewritten as

$$
Z_{\|, \mathrm{rw}}(\omega)=D \frac{|\omega|-i \omega}{\sqrt{|\omega|}}\left(1+C \omega^{2}\right)
$$

where

$$
C \equiv-\frac{2 b^{2}-r^{2}}{4 \beta^{2} \gamma^{2} c^{2}}
$$

and

$$
D \equiv \frac{Z_{0}}{4 \pi b c} \sqrt{\frac{2}{\mu_{0} \sigma}} .
$$

For $z<0$, we can evaluate (59) as follows:

$$
\begin{aligned}
W_{0}^{\prime}(z) & =\lim _{\epsilon \rightarrow 0} \frac{1}{2 \pi} \int_{-\infty}^{\infty} D \frac{|\omega|-i \omega}{\sqrt{|\omega+i \epsilon|}}\left(1+C \omega^{2}\right) e^{i \omega z / v} d \omega \\
& =\lim _{\epsilon \rightarrow 0} \frac{1}{\pi} \operatorname{Re}\left[\int_{0}^{\infty} D \frac{\omega-i \omega}{\sqrt{\omega+i \epsilon}}\left(1+C \omega^{2}\right) e^{i \omega z / v} d \omega\right]=\frac{1}{\pi} \int_{0}^{\infty} D \frac{i-1}{\sqrt{-i x}} x\left(1-C x^{2}\right) e^{-x|z| / v} d x \\
& =\frac{\sqrt{2}}{\pi} \int_{0}^{\infty} D \sqrt{x}\left(1-C x^{2}\right) e^{-x|z| / v} d x=\frac{\sqrt{2}}{\pi} \frac{D \sqrt{\pi}}{2(|z| / v)^{3 / 2}}\left(1-C \frac{15}{4(|z| / v)^{2}}\right) .
\end{aligned}
$$

In (63), we have first performed the substitution $\omega \rightarrow(-i x)$ and, thereafter, chosen the root $\sqrt{-i}=(1-i) / \sqrt{2}$ for reasons of continuity.

Inserting the expressions for $D$ and $C$, we finally get

$$
W_{0}^{\prime}(z, r) \approx \frac{\beta^{3 / 2} c Z_{0}}{4 \pi^{2}} \sqrt{\frac{\pi}{\mu_{0} \sigma c}} \frac{1}{2 b}[\operatorname{sgn}(z)-1]\left(\frac{1}{|z|^{3 / 2}}+\frac{15}{16} \frac{\left(2 b^{2}-r^{2}\right)}{\gamma^{2}|z|^{7 / 2}}\right)
$$

where the nonrelativistic correction term depends on the radial position $r$ of the test charge. Our convention is such that the wake field decelerates, if $W_{0}^{\prime}(z)>0$. Hence, the wake field is accelerating in the parameter regime considered here. We recall that in (55) we have expanded under the assumption $\left(\omega \delta_{\text {skin }} / c\right) \ll 1$, which corresponds to distances $|z| \gg 2 \chi b$, where $\chi \equiv 1 /\left(\sigma \mu_{0} b c\right)$ is a parameter defined by Chao [4]; we have also assumed, in (54), that $|\lambda b| \gg 1$, which is equivalent to $|z| \ll b / \chi$. Equation (64) should be accurate for the intermediate values of $z: 2 \chi b \ll|z| \ll b / \chi$.

In the ultrarelativistic limit, (64) reduces to the familiar form [see, e.g., Eq. (2.53) of [4]]

$$
W_{0}^{\prime}(z) \approx \frac{c^{2} \mu_{0}}{4 \pi^{2}} \sqrt{\frac{\pi}{\mu_{0} \sigma c}} \frac{1}{2 b}[\operatorname{sgn}(z)-1] \frac{1}{|z|^{3 / 2}} .
$$

\section{TRANSVERSE WAKE}

\section{A. The problem}

As before, we consider a current flowing in the longitudinal direction, with $j_{\varphi}=0, j_{r}=0$, and $\int j_{s} d r d \varphi=$ $v \lambda_{b}$. However, for computing the transverse wake, we now assume a source equal to a pure multipole of order $m$ with an effective strength $\lambda_{b} d^{m}$, where $d$ can be thought of as a net displacement between positive and negative charges. Every quantity, $V$, has the dependence $V=$ $\tilde{V} \exp (i m \varphi+i k s-i \omega t)=\tilde{V} \exp (i m \varphi+i k z)$ on $s, \varphi$, and $t$. In the following we consider only the dipole wake, for which $m= \pm 1$. For a normal dipole wake (as compared to a skew wake) the perturbation depends on the combination $\cos \varphi=\left(e^{i \varphi}+e^{-i \varphi}\right) / 2$.

\section{B. Wave equations in vacuum}

With a dipole moment as our source, all field components are nonzero, and, in particular, we can no longer assume that $A_{r}$ and $A_{\varphi}$ vanish. The wave equations (10) and (11) are still valid. However, in cylindrical coordinates the equations for the various components of the vector potential are no longer independent. Indeed, using the definition

$$
-\triangle \vec{A}=\vec{\nabla} \times(\vec{\nabla} \times \vec{A})-\vec{\nabla}(\vec{\nabla} \cdot \vec{A}),
$$

the wave equations in vacuum become 


$$
\begin{aligned}
& -\mu_{0} \tilde{j}_{r}=\frac{1}{r} \frac{\partial}{\partial r}\left[r \frac{\partial \tilde{A}_{r}}{\partial r}\right]-\frac{2 i m}{r^{2}} \tilde{A}_{\varphi}-\left[\frac{1+m^{2}}{r^{2}}+k_{r}^{2}\right] \tilde{A}_{r}, \quad-\mu_{0} \tilde{j}_{\varphi}=\frac{1}{r} \frac{\partial}{\partial r}\left[r \frac{\partial \tilde{A}_{\varphi}}{\partial r}\right]-\left[\frac{1+m^{2}}{r^{2}}+k_{r}^{2}\right] \tilde{A}_{\varphi}+\frac{2 i m}{r^{2}} \tilde{A}_{r} \\
& -\mu_{0} \tilde{j}_{s}=\frac{1}{r} \frac{\partial}{\partial r}\left[r \frac{\partial \tilde{A}_{s}}{\partial r}\right]-\left[\frac{m^{2}}{r^{2}}+k_{r}^{2}\right] \tilde{A}_{s}, \quad-\frac{\tilde{\rho}_{0}}{\epsilon_{0}}=\frac{1}{r} \frac{\partial}{\partial r}\left[r \frac{\partial \tilde{\phi}}{\partial r}\right]-\left[\frac{m^{2}}{r^{2}}+k_{r}^{2}\right] \tilde{\phi} .
\end{aligned}
$$

By introducing

$$
\begin{gathered}
A_{+} \equiv A_{r}+i A_{\varphi}, \\
A_{-} \equiv A_{r}-i A_{\varphi}, \\
j_{ \pm} \equiv j_{r} \pm j_{\varphi},
\end{gathered}
$$

the equations for $\tilde{A}_{r}$ and $\tilde{A}_{\varphi}$ can be decoupled:

$$
\frac{1}{r} \frac{\partial}{\partial r}\left(r \frac{\partial \tilde{A}_{ \pm}}{\partial r}\right)-\left(\frac{(1 \pm m)^{2}}{r^{2}}+k_{r}^{2}\right) \tilde{A}_{ \pm}=-\mu_{0} j_{ \pm},
$$

where $A_{ \pm}=\tilde{A}_{ \pm} \exp (i k z)$, etc. Note that the original radial and azimuthal components of the vector potential are given by

$$
A_{r}=\frac{A_{+}+A_{-}}{2}, \quad A_{\varphi}=\frac{A_{+}-A_{-}}{2 i} .
$$

\section{General solution for dipole wake}

Outside the beam, but inside the vacuum, the solution for each component again is a superposition of two Bessel functions, i.e., for $m=1$,

$$
\begin{aligned}
& \tilde{A}_{+}^{(1)}=p_{+}^{(1)} I_{2}\left(k_{r} r\right)+q_{+}^{(1)} K_{2}\left(k_{r} r\right), \\
& \tilde{A}_{-}^{(1)}=p_{-}^{(1)} I_{0}\left(k_{r} r\right)+q_{-}^{(1)} K_{0}\left(k_{r} r\right),
\end{aligned}
$$

$$
\begin{gathered}
\tilde{A}_{s}^{(1)}=p_{s}^{(1)} I_{1}\left(k_{r} r\right)+q_{s}^{(1)} K_{1}\left(k_{r} r\right), \\
\phi=p_{0}^{(1)} I_{1}\left(k_{r} r\right)+q_{0}^{(1)} K_{1}\left(k_{r} r\right),
\end{gathered}
$$

and, for $m=-1$,

$$
\begin{gathered}
\tilde{A}_{+}^{(-1)}=p_{+}^{(-1)} I_{0}\left(k_{r} r\right)+q_{+}^{(-1)} K_{0}\left(k_{r} r\right), \\
\tilde{A}_{-}^{(-1)}=p_{-}^{(-1)} I_{2}\left(k_{r} r\right)+q_{-}^{(-1)} K_{2}\left(k_{r} r\right), \\
\tilde{A}_{s}^{(-1)}=p_{s}^{(-1)} I_{1}\left(k_{r} r\right)+q_{s}^{(-1)} K_{1}\left(k_{r} r\right), \\
\phi=p_{0}^{(-1)} I_{1}\left(k_{r} r\right)+q_{0}^{(-1)} K_{1}\left(k_{r} r\right) .
\end{gathered}
$$

For a perturbation with $\varphi$ dependence $\cos \varphi=\left(e^{i \varphi}+\right.$ $\left.e^{-i \varphi}\right) / 2$, by symmetry, we have $p_{0}^{(-1)}=p_{0}^{(1)}, p_{s}^{(-1)}=p_{s}^{(1)}$, $q_{0}^{(-1)}=q_{0}^{(1)}, \quad q_{s}^{(-1)}=q_{s}^{(1)}, p_{+}^{(-1)}=p_{-}^{(1)}, \quad p_{-}^{(-1)}=p_{+}^{(1)}$, $q_{+}^{(-1)}=q_{-}^{(1)}$, and $q_{-}^{(-1)}=q_{+}^{(1)}$. This gives

$$
A_{r}=\frac{A_{r}^{(1)}+A_{r}^{(-1)}}{2}, \quad A_{\varphi}=\frac{A_{\varphi}^{(1)}+A_{\varphi}^{(-1)}}{2}, \cdots .
$$

With (72), we finally obtain

$$
\begin{aligned}
& A_{r}=\left(p_{+} I_{2}+q_{+} K_{2}+p_{-} I_{0}+q_{-} K_{0}\right) \cos \varphi e^{i k z}, \quad A_{\varphi}=\left(p_{+} I_{2}+q_{+} K_{2}-p_{-} I_{0}-q_{-} K_{0}\right) \sin \varphi e^{i k z}, \\
& A_{s}=\left(p_{s} I_{1}+q_{s} K_{1}\right) \cos \varphi e^{i k z}, \quad \phi=\left(p_{0} I_{1}+q_{0} K_{1}\right) \cos \varphi e^{i k z},
\end{aligned}
$$

where the argument of the Bessel functions is $\left(k_{r} r\right)$, and we have dropped the superindex "(1)" of all coefficients.

The Lorentz condition (9) relates these coefficients as

$$
\begin{array}{r}
\frac{k_{r}}{2}\left[p_{+} I_{1}\left(k_{r} r\right)-q_{+} K_{1}\left(k_{r} r\right)\right]+\frac{k_{r}}{2}\left[p_{-} I_{1}\left(k_{r} r\right)-q_{-} K_{1}\left(k_{r} r\right)\right]+i k\left[p_{s} I_{1}\left(k_{r} r\right)+q_{s} K_{1}\left(k_{r} r\right)\right]+ \\
\left(-i \frac{\omega}{c^{2}}+\mu_{0} \sigma\right)\left[p_{0} I_{1}\left(k_{r} r\right)+q_{0} K_{1}\left(k_{r} r\right)\right]=0
\end{array}
$$

which yields the two equations

$$
\begin{aligned}
& \frac{k_{r}}{2} p_{+}+\frac{k_{r}}{2} p_{-}+i k p_{s}-i \frac{\omega}{c^{2}} p_{0}+\mu_{0} \sigma p_{0}=0, \\
& -\frac{k_{r}}{2} q_{+}-\frac{k_{r}}{2} q_{-}+i k q_{s}-i \frac{\omega}{c^{2}} q_{0}+\mu_{0} \sigma q_{0}=0 .
\end{aligned}
$$

We still have some gauge freedom. Namely, similar to the longitudinal case, we can add the gradient of a scalar gauge function $\chi$ to $\vec{A}$. For example, if $\sigma=0$, using the gauge function $\chi=a I_{1}\left(k_{r} r\right) \cos \varphi \exp (i k z)$ we may transform the vector potential as $\vec{A}^{\prime}=\vec{A}-\vec{\nabla} \chi$ and $\phi^{\prime}=\phi+$ $\partial \chi / \partial t$. The Lorentz condition is preserved, since $\left[-c^{2} \vec{\nabla}\right.$. $\left.(\vec{\nabla} \chi)+\partial^{2} \chi / \partial t^{2}\right]=0$. Choosing $a=\left(p_{-}+p_{+}\right) / k_{r}$, we can achieve

$$
p_{-}=-p_{+},
$$

which can be verified by employing the relations $I_{1}^{\prime}(z)=$ $I_{0}(z)-I_{1}(z) / z$ and $(-2 / z) I_{1}(z)+I_{0}(z)=I_{2}(z)$. Inside the vacuum chamber, where $\sigma=0$, the Lorentz condition 
(83) then simplifies to

$$
p_{0}=\frac{c^{2} k}{\omega} p_{s}
$$

\section{Source boundary}

To determine the unknown coefficients, we proceed in analogy to the longitudinal case. We first consider the source terms. The current in the transverse direction is zero,

$$
j_{+}=j_{-}=j_{r}=j_{\varphi}=0,
$$

so that we are left with a longitudinal dipole current $j_{s}$ and a dipole charge $\rho$. The dipole-moment Green function for the right-hand sides of Eqs. (67) is $1 /(2 \pi r)$. In addition, it follows from the free-space solution for a 2dimensional pointlike dipole moment of strength $\left(\lambda_{b} d\right)$, that for decreasing distances to the origin the scalar potential should approach

$$
\phi \underset{r \rightarrow 0}{\rightarrow}-\frac{\lambda_{b} d}{2 \pi \epsilon_{0} r} \exp (i k z) \cos \varphi
$$

and the longitudinal vector potential should approach

$$
A_{s} \underset{r \rightarrow 0}{\rightarrow}-\frac{\mu_{0} v \lambda_{b} d}{2 \pi r} \exp (i k z) \cos \varphi .
$$

For small argument, the 1st order Bessel function $K_{1}\left(k_{r} r\right)$ expands as $1 /\left(k_{r} r\right)$. By equating the asymptotic solutions with the singular behavior of $K_{1}$, in the expressions for $A_{s}$ and $\phi$, we thus find that

$$
\begin{gathered}
q_{s}=\frac{\mu_{0} \lambda_{b} v}{2 \pi}\left(d k_{r}\right), \\
q_{0}=\frac{c^{2} k}{\omega} q_{s}, \\
q_{+}=0, \\
q_{-}=0 .
\end{gathered}
$$

Taking account of these simplifications, the potentials inside the beam pipe become

$$
\begin{aligned}
A_{r} & =p_{+}\left(I_{2}-I_{0}\right) \cos \varphi e^{i k z}, \quad A_{\varphi}=p_{+}\left(I_{2}+I_{0}\right) \sin \varphi e^{i k z}, \\
A_{s} & =\left(p_{s} I_{1}+q_{s} K_{1}\right) \cos \varphi e^{i k z}, \\
\phi & =\frac{c^{2} k}{\omega}\left(p_{s} I_{1}+q_{s} K_{1}\right) \cos \varphi e^{i k z},
\end{aligned}
$$

where $q_{s}$ is given in (90). Two coefficients are still unknown, namely $p_{+}$and $p_{s}$.

\section{E. Wall boundary}

As for the longitudinal case, we invoke the wall boundaries to determine the remaining two coefficients.
Thanks to $\vec{j}=\sigma \vec{E}$ inside the metal the equations for the potentials are the same as in vacuum, (67), except that $k_{r}^{2}$ is replaced by $\lambda^{2}$ defined in (32):

$$
\lambda^{2} \equiv \lambda_{0}^{2}+k_{r}^{2}=-\left(i \mu_{0} \sigma \omega-k_{r}^{2}\right)
$$

The general solutions for the potentials are as given in Eq. (82), only that the arguments of the Bessel functions are now $(\lambda r)$. We mark the coefficients for the solution inside the metal by the superindex " $w$." The coefficients of the Bessel functions $I_{k}$ are all zero, because these functions diverge for increasing argument. We can again apply a gauge transformation, this time to ensure $q_{+}^{w}=$ $-q_{-}^{w}$, which is achieved by the gauge function $\chi=$ $-\left(q_{+}^{w}+q_{-}^{w}\right) K_{1}(\lambda r) / \lambda \exp (i k z) \cos \varphi$, employing the relation $(2 / z) K_{1}(z)+K_{0}(z)=K_{2}(z)$. Then the potentials in the wall are

$A_{r}^{w}=q_{+}^{w}\left(K_{2}-K_{0}\right) \cos \varphi e^{i k z}, \quad A_{\varphi}^{w}=q_{+}^{w}\left(K_{2}+K_{0}\right) \sin \varphi e^{i k z}$, $A_{s}^{w}=q_{s}^{w} K_{1} \cos \varphi e^{i k z}, \quad \phi^{w}=\frac{1}{\frac{\omega}{k c^{2}}+i \frac{\mu_{0} \sigma}{k}} q_{s}^{w} K_{1} \cos \varphi e^{i k z}$,

where the argument of the Bessel functions is now $(\lambda r)$, and we have used the Lorentz condition (84)

$$
q_{s}^{w}=\left[\frac{\omega}{k c^{2}}+i \frac{\mu_{0} \sigma}{k}\right] q_{0}^{w}
$$

Now, we have four unknowns: $q_{s}^{w}$ and $q_{+}^{w}$ inside the wall, plus $p_{+}$and $p_{s}$ for the solution inside the chamber. These coefficients are determined by field matching at the chamber wall.

The longitudinal and azimuthal field components both inside the chamber wall and in the inner vacuum are related to the potentials via

$$
\begin{gathered}
B_{\varphi}=\left(i k \tilde{A}_{r}-\frac{\partial}{\partial r} \tilde{A}_{s}\right) \cos \varphi e^{i k z} \\
B_{s}=\left(\frac{1}{r} \tilde{A}_{r}+\frac{\partial \tilde{A}_{\varphi}}{\partial r}+\frac{\tilde{A}_{\varphi}}{r}\right) \sin \varphi e^{i k z} \\
B_{r}=\left(-\frac{1}{r} \tilde{A}_{s}-i k \tilde{A}_{\varphi}\right) \sin \varphi e^{i k z} \\
E_{\varphi}=\left(\frac{1}{r} \tilde{\phi}+i \omega \tilde{A}_{\varphi}\right) \sin \varphi e^{i k z} \\
E_{s}=\left(-i k \tilde{\phi}+i \omega \tilde{A}_{s}\right) \cos \varphi e^{i k z} \\
E_{r}=\left(-\frac{\partial \tilde{\phi}}{\partial r}+i \omega \tilde{A}_{r}\right) \cos \varphi e^{i k z}
\end{gathered}
$$

Requiring continuity of $E_{s}, E_{\varphi}, B_{\varphi}$, and $B_{s}$, at the chamber wall leads to the four constraints: 


$$
\begin{gathered}
-i \frac{k_{r}^{2}}{k_{0}}\left[p_{s} I_{1}\left(k_{r} b\right)+q_{s} K_{1}\left(k_{r} b\right)\right]+\left(\frac{i k_{0} \lambda^{2}}{k_{0}^{2}-\lambda_{0}^{2}}\right) q_{s}^{w} K_{1}(\lambda b)=0 \\
\frac{k}{b}\left(p_{s} I_{1}\left(k_{r} b\right)+q_{s} K_{1}\left(k_{r} b\right)-\frac{k_{0}^{2}}{k_{0}^{2}-\lambda_{0}^{2}} q_{s}^{w} K_{1}(\lambda b)\right)-i k_{0}^{2}\left[q_{+}^{w}\left(K_{2}(\lambda b)+K_{0}(\lambda b)\right)-p_{+}\left(I_{2}\left(k_{r} b\right)+I_{0}\left(k_{r} b\right)\right)\right]=0 \\
i k p_{+}\left[I_{2}\left(k_{r} b\right)-I_{0}\left(k_{r} b\right)\right]-p_{s} \frac{k_{r}}{2}\left[I_{0}\left(k_{r} b\right)+I_{2}\left(k_{r} b\right)\right]+\frac{k_{r}}{2} q_{s}\left[K_{0}\left(k_{r} b\right)+K_{2}\left(k_{r} b\right)\right]- \\
i k q_{+}^{w}\left[K_{2}(\lambda b)-K_{0}(\lambda b)\right]-q_{s}^{w} \frac{\lambda}{2}\left[K_{0}(\lambda b)+K_{2}(\lambda b)\right]=0 \\
\frac{1}{b} p_{+}\left[I_{2}\left(k_{r} b\right)-I_{0}\left(k_{r} b\right)\right]+p_{+} k_{r}\left[I_{2}^{\prime}\left(k_{r} b\right)+I_{1}\left(k_{r} b\right)\right]+\frac{p_{+}}{b}\left[I_{2}\left(k_{r} b\right)+I_{0}\left(k_{r} b\right)\right]-\frac{1}{b} q_{+}^{w}\left[K_{2}(\lambda b)-\right. \\
\left.K_{0}(\lambda b)\right]-q_{+}^{w} \lambda\left[K_{2}^{\prime}(\lambda b)-K_{1}(\lambda b)\right]-\frac{1}{b} q_{+}^{w}\left[K_{2}(\lambda b)+K_{0}(\lambda b)\right]=0,
\end{gathered}
$$

where use has been made of

$$
\begin{gathered}
I_{1}^{\prime}(z)=\frac{1}{2}\left[I_{0}(z)+I_{2}(z)\right], \\
K_{1}^{\prime}(z)=-\frac{1}{2}\left[K_{0}(z)+K_{2}(z)\right] .
\end{gathered}
$$

The magnetic field component $B_{r}$ is also continuous at the wall, if

$$
-\frac{1}{b}\left[p_{s} I_{1}\left(k_{r} b\right)+q_{s} K_{1}\left(k_{r} b\right)\right]-i k p_{+}\left[I_{2}\left(k_{r} b\right)+I_{0}\left(k_{r} b\right)\right]+\frac{1}{b} q_{s}^{w} K_{1}(\lambda b)+i k q_{+}^{w}\left[K_{2}(\lambda b)+K_{0}(\lambda b)\right]=0 .
$$

This condition is automatically fulfilled, if $E_{s}$ and $E_{\varphi}$ are continuous, as specified in Eqs. (104) and (105).

Solving Eqs. (104)-(107) for the unknown $p_{s}$, we obtain a rather complex expression, which we write as

$$
\frac{p_{s}}{q_{s}}=\frac{N}{D}
$$

where $N$ and $D$, respectively, denote the numerator and denominator. In the limit $|\lambda b| \gg 1$, we can expand the Bessel functions for the solution inside the wall,

$$
K_{i}(\lambda b) \approx \sqrt{\frac{\pi}{2 \lambda b}} e^{-\lambda b}, \quad \text { for } i=0, \ldots, 3,
$$

and in this limit the numerator and denominator are given by

$$
\begin{aligned}
N \approx & 2 b k_{0}^{2} k_{r}^{3} I_{2} K_{0}+2 b^{2} k_{0}^{2} k_{r}^{3} \lambda I_{2} K_{0}+2 b k_{0}^{2} k_{r} \lambda^{2} I_{2} K_{0}+2 b^{2} k_{0}^{2} k_{r} \lambda \lambda_{0}^{2} I_{2} K_{0}+b^{2} k_{0}^{2} k_{r}^{4} I_{3} K_{0}+b^{2} k_{0}^{2} k_{r}^{2} \lambda_{0}^{2} I_{3} K_{0}-4 b^{2} k_{0}^{2} k_{r}^{4} I_{2} K_{1} \\
& -4 b k_{0}^{2} k_{r}^{2} \lambda I_{2} K_{1}+4 k_{0}^{2} \lambda_{0}^{2} I_{2} K_{1}+4 k_{r}^{2} \lambda_{0}^{2} I_{2} K_{1}-4 b^{2} k_{0}^{2} k_{r}^{2} \lambda_{0}^{2} I_{2} K_{1}+4 b^{2} k_{r}^{4} \lambda_{0}^{2} I_{2} K_{1}-4 b k_{0}^{2} \lambda \lambda_{0}^{2} I_{2} K_{1}+4 b^{2} k_{r}^{2} \lambda_{0}^{4} I_{2} K_{1} \\
& -2 b^{2} k_{0}^{2} k_{r}^{3} \lambda I_{3} K_{1}+2 b^{2} k_{r}^{3} \lambda \lambda_{0}^{2} I_{3} K_{1}+2 b k_{0}^{2} k_{r}^{3} I_{2} K_{2}+2 b^{2} k_{0}^{2} k_{r}^{3} \lambda I_{2} K_{2}+2 b k_{0}^{2} k_{r} \lambda_{0}^{2} I_{2} K_{2}+2 b^{2} k_{0}^{2} k_{r} \lambda_{0}^{2} I_{2} K_{2}+b^{2} k_{0}^{2} k_{r}^{4} I_{3} K_{2} \\
& +b^{2} k_{0}^{2} k_{r}^{2} \lambda_{0}^{2} I_{3} K_{2}+3 b^{2} k_{r}^{2} I_{1}\left[k_{0}^{2}\left(k_{r}^{2}+\lambda_{0}^{2}\right) K_{0}++2 k_{r} \lambda\left(\lambda_{0}^{2}-k_{0}^{2}\right) K_{1}+k_{0}^{2}\left(k_{r}^{2}+\lambda_{0}^{2}\right) K_{2}\right] \\
& -2 I_{0}\left[-b k_{0}^{2} k_{r}(b \lambda-1)\left(k_{r}^{2}+\lambda_{0}^{2}\right) K_{0}+2\left\{\left(k_{0}^{2}+k_{r}^{2}\right) \lambda_{0}^{2}-b k_{0}^{2} \lambda\left(k_{r}^{2}+\lambda_{0}^{2}\right)+b^{2} k_{r}^{2}\left(k_{0}^{2}-\lambda_{0}^{2}\right)\left(k_{r}^{2}+\lambda_{0}^{2}\right)\right\} K_{1}\right. \\
& \left.\quad-b k_{0}^{2} k_{r}(-1+b \lambda)\left(k_{r}^{2}+\lambda_{0}^{2}\right) K_{2}\right], \\
& \\
& \begin{array}{l}
D \approx \\
\quad
\end{array} \\
& \quad+k_{0}^{2} k_{r}(b \lambda-1)\left(k_{r}^{2}+\lambda_{1}^{2}\right) I_{0}^{2}+6 b^{2} k_{r}^{3} \lambda\left(k_{0}^{2}-\lambda_{0}^{2}\right) I_{1}^{2}+b k_{0}^{2} k_{r}\left(\lambda_{0}^{2}+k_{r}^{2}\right) I_{2}\left[2(1+b \lambda) I_{2}+b k_{r} I_{3}\right] \\
& +I_{0}\left[\left\{4\left(k_{0}^{2}+k_{r}^{2}\right) \lambda_{0}^{2}-4 b k_{0}^{2} \lambda\left(k_{r}^{2}+\lambda_{0}^{2}\right)+b^{2} k_{r}^{2}\left(7 k_{0}^{2}-4 \lambda_{0}^{2}\right)\left(k_{r}^{2}+\lambda_{0}^{2}\right)\right\} I_{1}+b^{2} k_{0}^{2} k_{r}\left(k_{r}^{2}+\lambda_{0}^{2}\right)\left(4 \lambda I_{2}+k_{r} I_{3}\right)\right],
\end{aligned}
$$

where all remaining Bessel functions $K_{i}$ and $I_{i}(i=0, \ldots, 3)$ are evaluated at the argument $k_{r} b$. 
The longitudinal vector potential $A_{s}$ in the vacuum around the beam now follows from (94) as

$$
A_{s}=\left(\frac{N}{D} I_{1}\left(k_{r} r\right)+K_{1}\left(k_{r} r\right)\right) q_{s} \cos \varphi e^{i k z},
$$

where $q_{s}$ was determined in (90).

Unlike the other five field components, the radial electric field is not continuous. Its limiting values when approaching $r=b$ either from smaller or larger values of $r$ can be obtained from (91), (94), (103), and (115). The general expressions are rather intricate and we omit them here. In the limit of a perfect conductor $(\sigma \rightarrow \infty)$ and of a relativistic beam $\left(k_{r} \rightarrow 0\right)$, the expressions for the radial field greatly simplify, however, and the change in the radial electric field at the chamber wall due to the induced surface charge equals

$$
\left.E_{r}(r)\right|_{r \rightarrow b^{+}}-\left.E_{r}(r)\right|_{r \rightarrow b^{-}} \approx-\frac{\lambda d}{\pi \epsilon_{0} b^{2}} e^{i k z} \cos \varphi,
$$

which is a well-known expression [e.g., Eq. (1.8) in [4]]. In this limit the radial field is zero outside the wall boundary, or $\left.E_{r}(r)\right|_{r \rightarrow b^{+}} \rightarrow 0$.

\section{F. Impedance}

The vector potential (115) yields the longitudinal electric field via (94) and (102),

$$
E_{s}=-i \frac{c^{2} k_{r}^{2}}{\omega} \tilde{A}_{s} \cos \varphi e^{i k z}
$$

Inserting the above expressions for $A_{s}, N$, and $D$, we can expand $E_{s}$ through first order in the skin depth $\left(\delta_{\text {skin }} \omega / c\right)$, to second order in the parameter $\left(k_{r} r\right)$ or $\left(k_{r} b\right)$, and to third order in the radial position $r$ [involving products of terms $\left(k_{r} r\right)$ and $\left.\left(k_{r} b\right)\right]$. This gives

$$
\begin{aligned}
E_{s} & \approx i q_{s} \cos \varphi e^{i k z}\left(\frac{k_{r}\left(r^{2}-b^{2}\right)}{b^{2} \omega r}+\delta_{\text {skin }} \frac{1+i \operatorname{sgn}(\omega)}{2} \frac{c^{2} \omega\left(4-b^{2} k_{r}^{2}+\frac{1}{2} k_{r}^{2} r^{2}\right) r}{2 b^{3} k_{r}}\right) \\
& =i \cos \varphi e^{i k z} \frac{\mu_{0} \lambda_{b} v k_{r} d}{2 \pi}\left(\frac{k_{r} c^{2}\left(r^{2}-b^{2}\right)}{b^{2} \omega r}+\delta_{\text {skin }} \frac{1+i \operatorname{sgn}(\omega)}{2} \frac{\omega\left(4-b^{2} k_{r}^{2}+\frac{1}{2} k_{r}^{2} r^{2}\right) r}{2 b^{3} k_{r}}\right) .
\end{aligned}
$$

The longitudinal impedance of the dipole mode is defined as

$$
Z_{\|}^{(1)}(\omega)=-\frac{E_{s}}{\lambda_{b} d v r \cos \varphi e^{i k z}},
$$

which yields

$$
\begin{aligned}
Z_{\|}^{(1)}(\omega) & \approx-i \frac{Z_{0}}{c}\left(\frac{c^{2} k_{r}^{2}\left(r^{2}-b^{2}\right)}{2 \pi b^{2} \omega r^{2}}+\delta_{\text {skin }} \frac{1+i \operatorname{sgn}(\omega)}{2} \frac{\omega\left(4-b^{2} k_{r}^{2}+\frac{1}{2} k_{r}^{2} r^{2}\right)}{4 \pi b^{3}}\right) \\
& =-i Z_{0} \frac{c k_{r}^{2}\left(r^{2}-b^{2}\right)}{2 \pi b^{2} \omega r^{2}}+[1-i \operatorname{sgn}(\omega)] \frac{\left(4-b^{2} k_{r}^{2}+\frac{1}{2} k_{r}^{2} r^{2}\right)}{4 \pi b^{3} \delta_{\text {skin }} \sigma}
\end{aligned}
$$

We identify the first term, which is independent of the skin depth as the space-charge impedance,

$$
Z_{\|, \mathrm{sc}}^{(1)}(\omega) \approx-i \frac{Z_{0}}{2 \pi c} \frac{c^{2} k_{r}^{2}}{\omega} \frac{\left(r^{2}-b^{2}\right)}{b^{2} r^{2}}
$$

Here, we tried to emphasize the similarity with Eq. (56) for the monopole space-charge impedance $Z_{\|, \text {sc }}$.

The other term is the longitudinal dipole-mode impedance for the resistive wall including the lowest-order nonrelativistic correction:

$$
Z_{\|, \mathrm{rw}}^{(1)}(\omega) \approx[1-i \operatorname{sgn}(\omega)] \frac{\left(4-b^{2} k_{r}^{2}+\frac{1}{2} k_{r}^{2} r^{2}\right)}{4 \pi b^{3} \delta_{\text {skin }} \sigma} .
$$

The transverse wake is related to the longitudinal field by the Panofsky-Wenzel theorem

$$
\frac{\partial}{\partial z}\left[E_{r}+(\vec{v} \times \vec{B})_{r}\right]=\frac{\partial E_{s}}{\partial r},
$$

which, if expressed as above, is valid as in the relativistic case, thanks to $\omega=v k$. Equation (123) implies

$$
\begin{aligned}
Z_{\perp, \mathrm{rw}}(\omega) & =\frac{Z_{\|, \mathrm{rw}}^{(1)}(\omega)}{k} \approx \frac{Z_{\|, \mathrm{rw}}^{(1)}(\omega)}{\sqrt{\omega^{2} / c^{2}+k_{r}^{2}}} \\
& \approx[1-i \operatorname{sgn}(\omega)] \frac{1}{\sqrt{\omega^{2} / c^{2}+k_{r}^{2}}} \frac{\left(4-b^{2} k_{r}^{2}+\frac{1}{2} k_{r}^{2} r^{2}\right)}{4 \pi b^{3} \delta_{\text {skin }} \sigma}, \\
& \approx \frac{1-i \operatorname{sgn}(\omega)}{\omega / c} \frac{\left(4-b^{2} k_{r}^{2}+\frac{1}{2} k_{r}^{2} r^{2}-2 k_{r}^{2} c^{2} / \omega^{2}\right)}{4 \pi b^{3} \delta_{\text {skin }} \sigma}
\end{aligned}
$$

which is our final result for the transverse resistive-wall impedance.

According to their definition (1), the longitudinal and transverse Green-function dipole wakes are related to the associated impedances via Fourier transforms as 


$$
\begin{aligned}
& W_{1, \|}(z, r)=\frac{1}{2 \pi} \int_{-\infty}^{\infty} d \omega e^{i \omega z / v} Z_{\|}^{(1)}(\omega, r), \\
& W_{1, \perp}(z, r)=\frac{-i}{2 \pi} \int_{-\infty}^{\infty} d \omega e^{i \omega z / v} Z_{\perp}^{(1)}(\omega, r),
\end{aligned}
$$

where we have made the dependences on $z, \omega$, and $r$ explicit. From relations (123) or (124) it follows that

$$
W_{1, \perp}(z, r)=\frac{\partial}{\partial r} \int\left[W_{1, \|}(z, r) r\right] d z
$$

We note that due to the nonlinear dependence on $r$, the longitudinal dipole wake $W_{1, \|}(z, r)$ is not simply the $z$ derivative of the transverse wake $W_{1, \perp}$ as in the ultrarelativistic case [4], but that an additional factor $r$ and additional derivative with respect to $r$ enter in (127). Our $r$-dependent wake functions $W_{1, \perp}$ and $W_{1, \|}$ are defined such that they yield the forces acting on a test particle through the same relations as in Ref. [4].

\section{G. Green-function wake}

The transverse resistive-wall impedance is strongly diverging at the origin. Therefore, to determine the transverse Green-function wake we start from the longitudinal dipole-mode impedance, and we first compute the longitudinal dipole Green-function wake per unit length as

$$
W_{1, \|}(z, r)=\frac{1}{2 \pi} \int_{-\infty}^{\infty} Z_{\|, \mathrm{rw}}^{(1)}(\omega) e^{i \omega z / v} d \omega
$$

The integral is already familiar from (63)

$$
W_{1, \|}(z)=\frac{1}{\sqrt{\pi}} \frac{1}{(|z| / v)^{3 / 2}}\left[1+\frac{15\left(b^{2}-\frac{1}{2} r^{2}\right)}{16|z|^{2} \gamma^{2}}\right] \frac{2}{\pi b^{3} \sqrt{\sigma / \mu_{0}}} \frac{(\operatorname{sgn}(z)-1)}{2} .
$$

As indicated above, we obtain the transverse Green-function wake $W_{1}(z)$ by integrating $\left[W_{1, \|}(z, r) r\right]$ with respect to $z$ and differentiating with respect to $r$ :

$$
W_{1, \perp}(z, r)=\frac{\partial}{\partial r} \int_{-\infty}^{z} W_{1, \|}(u, r) r d u=\beta^{3 / 2} c Z_{0} \frac{1}{\pi^{2}} \frac{1}{b^{3}} \sqrt{\frac{\pi}{c \mu_{0} \sigma}}\left[\frac{1}{|z|^{1 / 2}}+\frac{3}{8} \frac{b^{2}-\frac{3}{2} r^{2}}{\gamma^{2}|z|^{5 / 2}}\right] \frac{(\operatorname{sgn}(z)-1)}{2} .
$$

In the ultrarelativistic limit, this becomes

$$
W_{1, \perp}(z) \approx c Z_{0} \frac{1}{\pi^{2}} \frac{1}{4 b^{3}} \sqrt{\frac{\pi}{c \mu_{0} \sigma}} \frac{1}{|z|^{1 / 2}} \frac{(\operatorname{sgn}(z)-1)}{2},
$$

which agrees with the classical result [see, e.g., again Eq. (2.53) of [4]].

\section{COMPARISON WITH EARLIER RESULTS}

Equation (57) gives the longitudinal resistive-wall impedance including the nonrelativistic correction to first order in $\left(\delta_{\text {skin }} \omega / c\right)$ and to second order in $\left(k_{r} c / \omega\right)$ as

$$
Z_{\|, \mathrm{rw}}(\omega) \approx \frac{Z_{0}}{2 \pi c}\left[\frac{(\operatorname{sgn}(\omega)-i) \omega \delta_{\text {skin }}}{2 b}\left(1-\frac{k_{r}^{2}\left(2 b^{2}-r^{2}\right)}{4}\right)\right] .
$$

In Ref. [7] Al-khateeb et al. calculated the longitudinal resistive-wall impedance by a different approach and defined it via the energy loss of a uniform beam with transverse size $a$. We can identify the parameter $\sigma$ of [7] with our variable $k_{r}$. Also setting $a=r$, Al-khateeb's complete expression for the resistive impedance [Eqs. (33)-(35) in Ref. [7]], per unit length, is rewritten as

$$
\begin{aligned}
Z_{\|, \mathrm{rw}}^{\mathrm{Al}-\mathrm{khateeb}}(\omega)=\frac{Z_{0} \delta_{\text {skin }} \omega}{4 \pi b c}[ & \frac{\frac{4}{\left(k_{r} r\right)^{2}} I_{1}^{2}\left(k_{r} r\right)}{I_{0}^{2}\left(k_{r} b\right)-2 \alpha I_{0}\left(k_{r} b\right) I_{1}\left(k_{r} b\right)+2 \alpha^{2} I_{1}\left(k_{r} b\right)^{2}} \\
& \left.-i \frac{\frac{4}{\left(k_{r} r\right)^{2}} I_{1}^{2}\left(k_{r} r\right)\left[I_{0}\left(k_{r} b\right)-2 \alpha I_{1}\left(k_{r} b\right)\right]}{I_{0}\left(k_{r} b\right)\left(I_{0}^{2}\left(k_{r} b\right)-2 \alpha I_{0}\left(k_{r} b\right) I_{1}\left(k_{r} b\right)+2 \alpha^{2} I_{1}\left(k_{r} b\right)^{2}\right)}\right],
\end{aligned}
$$

where

$$
\alpha=\left(\omega^{2} \delta_{\text {skin }}\right) /\left(2 k_{r} c^{2}\right),
$$

and we have inverted the sign of the imaginary part. Expanding this expression as before to first order in $\left(\delta_{\text {skin }} \omega / c\right)$ and to second order in $\left(k_{r} c / \omega\right)$ we get

$$
Z_{\|, \mathrm{rw}}^{\mathrm{Al} \text {-khateeb }}(\omega) \approx \frac{Z_{0}}{2 \pi c}\left[\frac{(1-i) \omega \delta_{\text {skin }}}{2 b}\left(1-\frac{k_{r}^{2}\left(2 b^{2}-r^{2}\right)}{4}\right)+i \frac{b^{2} k_{r}^{2}}{4}\right],
$$

which differs from our expression (131) by the last imaginary term without a real counterpart. 
The expansion of the approximation (37) in Ref. [7] does not contain this term and it agrees with our (131). Hence, this formula is not consistent with the preceding (134) nor with Eqs. (33)-(35) of [7]. However, it is interesting to note that an alternative expression, namely, Eq. (41) in Ref. [7], which was derived by computing the power loss in the pipe wall, is equal to our result (131) in the limit considered here.

In Ref. [8], Gluckstern considered as source a narrow ring of radius $a$ and, calculating the energy loss over the distribution, for the nonrelativistic resistive-wall impedance he derived the expression [Eqs. (5.7) and (5.8) in [8]]

$$
\begin{aligned}
Z_{\|, \mathrm{rw}}^{\mathrm{Gluckstern}}(\omega)= & i \frac{Z_{0} I_{0}(k r a)^{2} k_{r}^{2} c}{2 \pi \omega} \\
& \times\left[\frac{K_{0}\left(k_{r} b\right)}{I_{0}\left(k_{r} b\right)}-\frac{K_{0}\left(b k_{r}+(1+i) \alpha K_{1}\left(b k_{r}\right)\right)}{I_{0}\left(b k_{r}-(1+i) \alpha I_{1}\left(b k_{r}\right)\right)}\right],
\end{aligned}
$$

where $\alpha$ was defined in (133). Expanding again to first order in $\left(\delta_{\text {skin }} \omega / c\right)$ and to second order in $\left(k_{r} c / \omega\right)$ and setting $a=r$, Gluckstern's formula reduces to

$$
Z_{\|, \mathrm{rw}}^{\mathrm{Gluckstern}}(\omega) \approx \frac{Z_{0}}{2 \pi c}\left[\frac{(1-i) \omega \delta_{\text {skin }}}{2 b}\left(1-\frac{k_{r}^{2}\left(2 b^{2}-2 r^{2}\right)}{4}\right)\right] \text {. }
$$

For $\omega>0$, this expression equals our Eq. (131) except for a factor 2 in the coefficient of the $r$-dependent term, which we attribute to a different definition of impedance. We note that Gluckstern's formula (135) does not include any unpaired imaginary component as is present in Alkhateeb's expression (132) or (134).

Gluckstern also computed the transverse nonrelativistic resistive-wall impedance, in this case considering an ideal dipole-moment source as we have done in Sec. IV. Gluckstern's expression [Eqs. (5.23) and (5.24) in [8]] can be written as

$$
Z_{\perp, \mathrm{rw}}^{\mathrm{Gluckstern}}(\omega)=\frac{v b(1-i) Z_{0} \delta_{\text {skin }}}{2 c} \frac{k_{r}^{2}}{4 \pi} \frac{1}{b^{2} I_{1}\left(b k_{r}\right)^{2}},
$$

and its expansion is

$$
Z_{\perp, \mathrm{rw}}^{\mathrm{Gluckstern}}(\omega) \approx \frac{(1-i) Z_{0} \delta_{\text {skin }}}{8 \pi b^{3}}\left(4-b^{2} k_{r}^{2}-2 k_{r}^{2} c^{2} / \omega^{2}\right),
$$

which, up to the order of expansion, agrees with our solution (124),

$$
Z_{\perp, \mathrm{rw}}(\omega) \approx \frac{1-i \operatorname{sgn}(\omega)}{\omega / c} \frac{\left(4-b^{2} k_{r}^{2}+\frac{1}{2} k_{r}^{2} r^{2}-2 \frac{k_{r}^{2} c^{2}}{\omega^{2}}\right)}{4 \pi b^{3} \delta_{\text {skin }} \sigma},
$$

if we evaluate the latter at amplitude $r=0$.

Finally, we note that the Green-function wake fields presented in [10] were incorrect. They were defined and computed by a Fourier transform with respect to $k$ rather than with respect to $\omega$, without taking into consideration that, in the general case, $k$ is not continuous, i.e., it does not assume the values between $-k_{0}$ and $+k_{0}$.

\section{LIMITS OF VALIDITY}

We have assumed the conductivity $\sigma$ to be a scalar quantity, independent of frequency. This is valid only for metals whose lattice is of cubic symmetry (e.g., $\mathrm{Al}, \mathrm{Cu}$, or $\mathrm{Fe}$ ), while for metals without this symmetry (e.g., Ti), the conductivity is a tensor [11]. In addition to changes introduced by the crystal structure, modifications to Ohm's law are also imposed by ac conductivity [12], anomalous skin effect [13], and magnetoresistance [11], none of which was included in our analysis. These latter effects become important at high frequency, for low temperature, or in a strong magnetic field. Our results are also modified, if the relative magnetic permeability or the relative dielectric constant differ from 1 . Other possible complications that were not considered in our treatment are related to the finite length of real beam-pipe components and to the relative velocity of the wake and the beam.

We first discuss the consequences of ac conductivity. At sufficiently high frequencies the conductivity $\sigma$ can no longer be treated as a constant $\sigma$. It varies with frequency as $[11,12]$

$$
\bar{\sigma}(\omega)=\frac{\sigma}{1-i \omega \tau_{\text {rel }}} .
$$

Table I lists relaxation times $\tau_{\text {rel }}$ for three typical metals at various temperatures [11] and the implied limits on the maximum frequency, beyond which the formulas presented in this paper must be modified. Note that the maximum frequency $\omega_{\max }$ listed in Table I is related to the minimum distance $z_{\min }$ for which the computed expression for the wake field remains valid via $z_{\min } \approx$ $c \beta / \omega_{\max }$. For example, at a typical value $\omega_{\max } \approx$ $10^{13} \mathrm{~s}^{-1}$ and for $\beta \approx 1$ the ac component of the conductivity becomes significant at distances below $30 \mu \mathrm{m}$.

The next limit we estimate is due to the anomalous skin effect. At low temperature and high frequencies, the mean free path of the electrons exceeds the frequencydependent classical skin depth, $\delta_{\text {skin }}$, in which case the surface resistivity deviates from the classical value. The theory of this "anomalous skin effect" was developed by a number of authors [13-16]. The classical formulas for the surface resistance cease to be valid, if [17]

$$
\alpha \equiv \frac{3}{4} \mu_{0} \omega(\rho l)^{2} \frac{1}{\rho^{3}} \geq 0.02,
$$

or if

$$
\omega \geq 0.027 \frac{\rho^{3}}{\mu_{0}(\rho l)^{2}} .
$$

Relevant parameters for the same three example materials at different temperatures are compiled in Table II. The anomalous skin effect can be a concern at low temperatures. For example, for $\omega_{\max } \approx 10^{11} \mathrm{~s}^{-1}$ and $\beta \approx 1$ 
TABLE I. Relaxation times [11] and implied frequency limits due to the onset of ac conductivity for three metals and temperatures.

\begin{tabular}{ccc}
\hline \hline Metal, $T$ & $\tau_{\text {rel }}\left(10^{-14} \mathrm{~s}\right)$ & $\omega_{\max }\left(10^{12} \mathrm{~s}^{-1}\right)$ \\
\hline $\mathrm{Cu}$ at $77 \mathrm{~K}$ & 21 & 4.8 \\
at $273 \mathrm{~K}$ & 37 & 2.7 \\
at $373 \mathrm{~K}$ & 53 & 1.9 \\
$\mathrm{Al}$ at $77 \mathrm{~K}$ & 6.5 & 15 \\
at $273 \mathrm{~K}$ & 0.80 & 125 \\
at $373 \mathrm{~K}$ & 0.55 & 182 \\
Fe at $77 \mathrm{~K}$ & 3.2 & 31 \\
at $273 \mathrm{~K}$ & 0.24 & 420 \\
at $373 \mathrm{~K}$ & $0.14 \mathrm{~K}$ & 714 \\
\hline \hline
\end{tabular}

the anomalous effect starts to appear at distances below $3 \mathrm{~mm}$.

The last frequency limit, induced by magnetoresistance, occurs in the presence of large magnetic fields. Specifically, magnetoresistance is noticeable, if the product of the angular cyclotron frequency and the relaxation time $\tau_{\text {rel }}$ of the metal approaches or exceeds 1 , i.e., if

$$
\frac{e B}{m_{e}} \tau_{\text {rel }} \geq 1
$$

Table III lists the implied limits on the magnetic field, beyond which our formulas are no longer valid. The table suggests that magnetoresistance might be important only for a few metals, possibly copper, and only at extremely low temperatures (a few $\mathrm{K}$ ) in a high magnetic field (several T); for an example, see [18].

Though we have assumed $\mu_{r}=1$, this is not a real limitation, as all the formulas derived in this report could be extended to $\mu_{r} \neq 1$ by the replacements $\sigma \rightarrow \sigma \mu_{r}$ and $k_{0}^{2} \rightarrow k_{0}^{2} \mu_{r}$.

All impedances and wake fields computed in this report refer to the steady -state established in an infinitely long beam pipe of thickness larger than the skin depth. For short beam line elements, local variations in the

TABLE II. Resistivity, product $(\rho l)$ [11], and implied frequency limits where the anomalous skin effect becomes important, for three metals and temperatures.

\begin{tabular}{cccc}
\hline \hline Metal, $T$ & $\rho(\mu \Omega \mathrm{cm})$ & $(\rho l)\left(10^{-16} \Omega \mathrm{m}^{2}\right)$ & $\omega_{\max }\left(10^{9} \mathrm{~s}^{-1}\right)$ \\
\hline $\mathrm{Cu}$ at $77 \mathrm{~K}$ & 0.2 & 7.1 & 0.3 \\
at $273 \mathrm{~K}$ & 1.56 & 7.1 & 160 \\
at $373 \mathrm{~K}$ & 2.24 & 7.1 & 480 \\
$\mathrm{Al}$ at $77 \mathrm{~K}$ & 0.3 & 4.3 & 3 \\
at $273 \mathrm{~K}$ & 2.45 & 4.3 & 1700 \\
at $373 \mathrm{~K}$ & 3.55 & 4.3 & 5200 \\
Fe at $77 \mathrm{~K}$ & 0.66 & 4.5 & 23 \\
at $273 \mathrm{~K}$ & 8.9 & 4.5 & $7.5 \times 10^{4}$ \\
at $373 \mathrm{~K}$ & 14.7 & 4.5 & $3.4 \times 10^{5}$ \\
\hline \hline
\end{tabular}

TABLE III. Limits on the magnetic field beyond which magnetoresistance must be taken into account for three metals and temperatures, using the relaxation times of Table I.

\begin{tabular}{cc}
\hline \hline Metal, $T$ & $B_{\max }(\mathrm{T})$ \\
\hline $\mathrm{Cu}$ at $77 \mathrm{~K}$ & 27 \\
at $273 \mathrm{~K}$ & 210 \\
at $373 \mathrm{~K}$ & 299 \\
$\mathrm{Al}$ at $77 \mathrm{~K}$ & 87 \\
at $273 \mathrm{~K}$ & 710 \\
at $373 \mathrm{~K}$ & 1032 \\
$\mathrm{Fe}$ at $77 \mathrm{~K}$ & 177 \\
at $273 \mathrm{~K}$ & 2366 \\
at $373 \mathrm{~K}$ & 4056 \\
\hline \hline
\end{tabular}

surface composition, metallic elements outside of the chamber, and the resulting 3-dimensional current "redistribution" may substantially alter the impedances and wake fields $[19,20]$.

Last, by invoking the exponential dependence $\exp (i k z-i \omega t)$, we have assumed that the harmful electric and magnetic fields constituting the wake propagate at the same speed as the beam. However, multibunch resistive-wall instabilities in a large storage ring can be driven by field components at much smaller phase velocity [21]. This possibility may be taken into account by introducing a generalized wake field, which takes wave number $k$ and frequency $\omega$ as two independent arguments [21]. The result is a different concept of nonrelativistic wake, namely, one that would affect even an ultrarelativistic beam. Such generalized nonrelativistic wake has not been addressed here.

\section{APPLICATIONS}

Typical parameters of several low-energy proton or ion accelerators are listed in Table IV. The abbreviated accelerator names in the top row of the table refer to the Accumulator Ring of the Spallation Neutron Source (SNS) [1], to the 3-GeV rapid-cycling synchrotron of the Japan Proton Accelerator Research Complex (J-PARC) [2], to the booster rings of the CERN Proton Synchrotron (PS booster) [3], and to an electron-cyclotron resonance (ECR) ion source (e.g., [22]). The energy decreases from SNS, over J-PARC and the PS booster to an ECR source.

TABLE IV. Example parameters.

\begin{tabular}{ccccc}
\hline \hline & SNS & J-PARC & PS booster & ECR \\
\hline$\gamma$ & 2.1 & 1.4 & 1.05 & 1.003 \\
$\sigma_{z}$ & $25 \mathrm{~m}$ & $12 \mathrm{~m}$ & $26 \mathrm{~m}$ & $100 \mathrm{~m}$ \\
$\sigma_{r}$ & $2 \mathrm{~cm}$ & $2 \mathrm{~cm}$ & $3 \mathrm{~mm}$ & $4 \mathrm{~mm}$ \\
$b$ & $8 \mathrm{~cm}$ & $12.5 \mathrm{~cm}$ & $30 \mathrm{~cm}$ & $3 \mathrm{~cm}$ \\
$b / \gamma$ & $0.038 \mathrm{~m}$ & $0.089 \mathrm{~m}$ & $0.286 \mathrm{~m}$ & $0.030 \mathrm{~m}$ \\
$Q N_{b}$ & $1.5 \times 10^{14}$ & $4 \times 10^{13}$ & $1.2 \times 10^{12}$ & $2 \times 10^{13}$ \\
\hline \hline
\end{tabular}




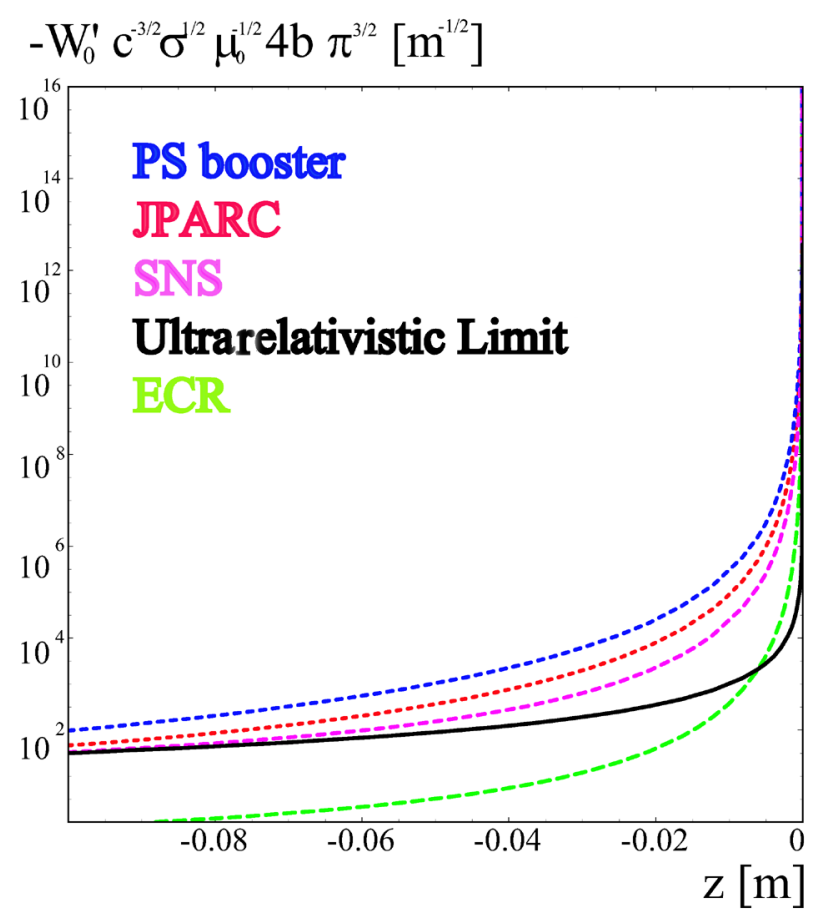

FIG. 1. (Color) Longitudinal wake $\left|W_{0}^{\prime}\right| 4 \pi^{3 / 2} b \sqrt{\sigma c / \mu_{0}} / c^{2}$ at $r=0$ vs distance $z$ in $m$, according to (64) (colored lines) for the examples of Table IV, and in the ultrarelativistic limit (65) (solid black line).

For each case we consider a stainless steel chamber with conductivity $\sigma=1.4 \times 10^{6}-\Omega^{-1} \mathrm{~m}^{-1}$. Figure 1 compares the nonrelativistic longitudinal wake functions at the center of the chamber for these four cases with the ultrarelativistic expression. Figure 2 presents an analogous picture for the transverse case. The two figures illustrate that the differences between the ultrarelativistic limit, (65) and (131), and the more accurate formulas, (64) and (131), are significant for $\gamma<3$ for all values of $z$ due to the multiplying factor $\beta^{3 / 2}$ in (64) and (131). Also, the additive nonrelativistic corrections, i.e., the terms inside the parentheses, are seen to become important for short distances $z$, specifically, for $z>-\sqrt{15 / 8} b / \gamma$ longitudinally, and for $z>-\sqrt{3 / 8} b / \gamma$ transversely.

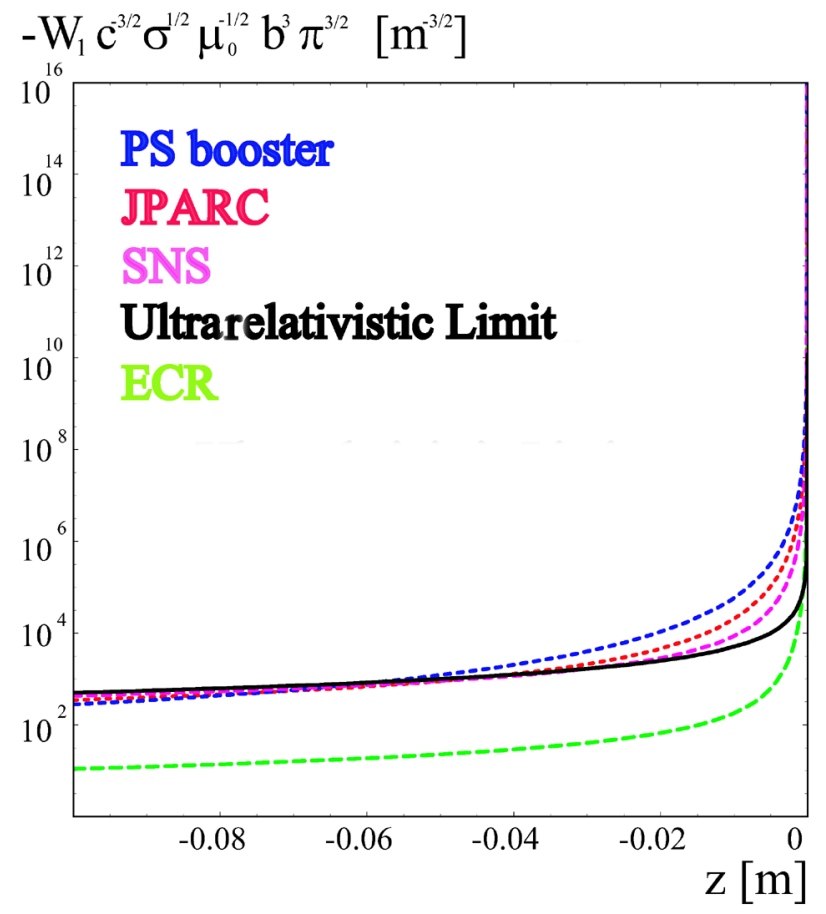

FIG. 2. (Color) Transverse resistive-wall wake $\left|W_{1, \perp}\right| \pi^{3 / 2} b^{3} \sqrt{\sigma c / \mu_{0}} / c^{2}$ vs distance $z$ in $m$, according to (131) (colored lines) for the examples of Table IV, and in the ultrarelativistic limit (131) (solid black line).

The deviations from the classical ultrarelativistic expression can amount to 1 or 2 orders of magnitude. They are largest for the PS booster and the ECR, i.e., for the examples with the lowest beam energy.

\section{SUMMARY}

We have derived the lowest-order nonrelativistic corrections to the longitudinal and transverse resistive-wall impedances and the associated Green-function wake fields for a beam passing through an infinite circular metallic beam pipe.

Our main results are as follows. The longitudinal impedance including nonrelativistic terms is given by (57)

$$
Z_{\|, \mathrm{rw}}(\omega) \approx \frac{Z_{0}}{2 \pi c}\left[\frac{(\operatorname{sgn}(\omega)-i) \omega \delta_{\text {skin }}}{2 b}\left(1-\frac{k_{r}^{2}\left(2 b^{2}-r^{2}\right)}{4}\right)\right],
$$

and the longitudinal Green-function wake by (64)

$$
W_{0}^{\prime}(z, r) \approx \frac{\beta^{3 / 2} c Z_{0}}{4 \pi^{2}} \sqrt{\frac{\pi}{\mu_{0} \sigma c}} \frac{1}{2 b}(\operatorname{sgn}(z)-1)\left(\frac{1}{|z|^{3 / 2}}+\frac{15}{16} \frac{\left(2 b^{2}-r^{2}\right)}{\gamma^{2}|z|^{7 / 2}}\right) .
$$

The transverse impedance, derived in (122), is

$$
Z_{\|, \mathrm{rw}}^{(1)}(\omega) \approx(1-i \operatorname{sgn}(\omega)) \frac{\left(4-b^{2} k_{r}^{2}+\frac{1}{2} k_{r}^{2} r^{2}\right)}{4 \pi b^{3} \delta_{\mathrm{skin}} \sigma},
$$

and the corresponding wake function, in (131), 


$$
W_{1, \perp}(z, r)=\beta^{3 / 2} c Z_{0} \frac{1}{\pi^{2}} \frac{1}{b^{3}} \sqrt{\frac{\pi}{c \mu_{0} \sigma}}\left[\frac{1}{|z|^{1 / 2}}+\frac{3}{8} \frac{b^{2}-\frac{3}{2} r^{2}}{\gamma^{2}|z|^{5 / 2}}\right] \frac{(\operatorname{sgn}(z)-1)}{2} .
$$

The expressions for the impedance are consistent with some, though not all, formulas previously derived. The Green-function wakes are here given explicitly for the first time.

The nonrelativistic formulas for the resistive-wall wake fields significantly deviate from those of the ultrarelativistic limit both at low energies, $\gamma<3$, and at short distances, $|z| / b<1 / \gamma$.

The expressions developed in this report cease to be valid for metal lattices without cubic symmetry, at high frequencies or in strong magnetic fields, i.e., whenever the response of the chamber material can no longer be described by Ohm's law with a constant scalar conductivity. Additional complications are expected to arise for metals with dielectric constants and magnetic permeabilities different from 1, for beam line elements of finite length where the electromagnetic fields do not reach a steady state, as well as in cases where wake fields propagating at phase velocities different from the beam velocity are important.

\section{ACKNOWLEDGMENTS}

We thank G. Rumolo and A. Fedotov for pointing us to Refs. [7,8] during the PAC2003 conference. We also thank D. Kaltchev for helpful discussions on complex integration. We acknowledge helpful discussions with F. Ruggiero and K. Yokoya, who brought Refs. [6,21], respectively, to our attention.

[1] J. Wei, in Proceedings of the Workshop on Space Charge Physics in High Intensity Hadron Rings, Shelter Island, New York, 1998, AIP Conf. Proc. No. 448 (AIP, New York, 1998), p. 152.

[2] Y. Yamazaki et al., KEK REPORT No. 2002-13, JAERI Report No. TECH-2003-044, 2003.

[3] M. Benedikt et al., in Proceedings of the EPAC 2000, Vienna (European Physical Society Interdivisional Group on Accelerators, Geneva, 2000).
[4] A. Chao, Physics of Collective Beam Instabilities in High-Energy Accelerators (Wiley, New York, 1993).

[5] B. Zotter and S. Heifets, Impedances and Wakes in High-Energy Particle Accelerators (World Scientific, Singapore, 1998).

[6] P. Morton, V. K. Neil, and A. Sessler, J. Appl. Phys. 37, 3875 (1966).

[7] A. M. Al-khateeb, O. Boine-Frankenheim, I. Hofmann, and G. Rumolo, Phys. Rev. E 63, 026503 (2001).

[8] R. L. Gluckstern, CERN Report No. 2000-011, 2000.

[9] M. Abramowitz and I. A. Stegun, in Handbook of Mathematical Functions with Formulas, Graphs, and Mathematical Tables, Natl. Bur. Stand., Appl. Math. No. 55 (U.S. GPO, Washington, DC, 1970).

[10] F. Zimmermann and K. Oide, in Proceedings of the PAC2003, Portland (IEEE, Piscataway, NJ, 2003).

[11] N.W. Ashcroft and N. D. Mermin, Solid State Physics (Holt, Rinehart, and Winston, New York (1976).

[12] K. Bane and M. Sands, in Proceedings of the Micro Bunches Workshop, Upton, NY, 1995, AIP Conf. Proc. No. 367 (AIP, New York, 1996).

[13] G. E. H. Reuter and E. H. Sondheimer, Proc. R. Soc. London, Ser. A 195, 336 (1948).

[14] H. London, Proc. R. Soc. London, Ser. A 176, 522 (1940).

[15] A. B. Pippard, Proc. R. Soc. London, Ser. A 191, 385 (1947).

[16] R. G. Chambers, Proc. R. Soc. London, Ser. A 215, 481 (1952).

[17] J. T. Rogers et al., Appl. Phys. Lett. 52, 26 (1988).

[18] W. Chou and F. Ruggiero, CERN LHC Project Note No. 2 (SL/AP), 1995.

[19] F. Caspers (private communication).

[20] L. Vos, D. Brandt, F. Caspers, A. Mostacci, L. Rinolfi, and H. Tsutsui, CERN-AB-MD-Note No. 2003-002-MD, 2003.

[21] M. M. Karliner, N.V. Mityanina, D. G. Myakishev, and V.P. Yakovlev, in Proceedings of the EPAC, Sitges, Barcelona, 1996 (IOP, Bristol, 1996), p. 1247.

[22] C. E. Hill et al., in Proceedings of the 12th Chamonix LHC Performance Workshop, 2003 (CERN Report No. CERN-AB-2003-008 ADM, 2003). 